\title{
Mast Cells as an Indicator and Prognostic Marker in Molecular Subtypes of Breast Cancer
}

\author{
ECATERINA CARPENCO ${ }^{1,2}$, RALUCA AMALIA CEAUŞU ${ }^{3,4}$, ANCA MARIA CIMPEAN $^{3,4}$, PUŞA NELA GAJE $^{3,4}$, \\ LILIAN S,APTEFRAŢI ${ }^{1}$, VEACESLAV FULGA ${ }^{1,2}$, VALERIU DAVID ${ }^{1,2}$ and MARIUS RAICA ${ }^{3,4}$ \\ ${ }^{1}$ Department of Histology, Cytology and Embryology, \\ Nicolae Testemițanu State University of Medicine and Pharmacy, Chișinău, Republic of Moldova; \\ ${ }^{2}$ Laboratory of Morphology, Nicolae Testemițanu State University of Medicine and Pharmacy, \\ Chișinău, Republic of Moldova; \\ ${ }^{3}$ Department of Microscopic Morphology/Histology, ${ }^{4}$ Angiogenesis Research Center Timișoara, \\ Victor Babeș University of Medicine and Pharmacy, Timișoara, Romania
}

\begin{abstract}
Background/Aim: Mast cells (MCs) represent the most controversial non-malignant element of the tumor microenvironment. Our aim was to study how MCs density and distribution (intratumoral-MCit versus peritumoral-MCpt) relate to tumor grade and molecular subtypes. Materials and Methods: MCs tryptase immunohistochemistry was performed on 80 cases of breast carcinomas. Results: For Luminal A tumors, a partial correlation was detected between MCit and progesterone receptor $(P R)(p=0.005)$. Luminal B tumors showed a significant correlation between MCpt and age $(p=0.009)$, estrogen receptor (ER) $(p=0.017)$ and $P R(p=0.035)$. MCit and MCpt were strongly interrelated in this subtype $(p=0.002)$ and in triplenegative breast cancers ( $p=0.002)$. In HER2 subtype, MCpt tumors were significantly correlated with HER2 ( $p=0.044)$. In G2 tumors, MCpt correlated with ER $(p=0.015)$ and $P R$ $(p=0.038)$ while in G3 tumors ER correlated with both MCit ( $p=0.009)$ and MCpt $(p=0.000487)$ tumors. Conclusion: MCs dynamics are strongly influenced by hormone receptors and HER2 status. MCit increased in aggressive tumor types and is a worse prognostic factor.
\end{abstract}

Breast cancer is the most common type of cancer in women and the second most common cancer overall. Over 2 million

This article is freely accessible online.

Correspondence to: Anca Maria Cimpean, Department of Microscopic Morphology/Histology, Angiogenesis Research Center, Victor Babeș University of Medicine and Pharmacy, 300041, Piața Eftimie Murgu nr.2, Timișoara, Romania. Tel: +40 720060955, e-mail: ancacimpean1972@yahoo.com

Key Words: Breast cancer, hormone receptors, mast cell, prognosis, tryptase, tumor grade. new cases of breast cancer appeared in 2018, and its incidence and mortality are rapidly growing worldwide (1). Breast cancer is a complex and heterogeneous disease in terms of microscopic features, therapeutic response, spreading to distant sites, and patients' outcomes (2). A possible explanation could be, in part, that we still lack a complete picture of the biological heterogeneity of breast cancer with respect to molecular changes and cellular composition. Importantly, this complexity is not entirely reflected by the main clinical parameters (age, lymph node status, tumor size, histological grade) and molecular markers. Molecular classification, introduced by Prat and Perou, is routinely used in the clinic to stratify patients for prognostic predictions and select treatments; however, it does not perfectly describe all the entities and there are still many debated issues $(2,3)$.

Cancer research is focused mainly on malignant cells, while non-malignant components of the tumor microenvironment are less investigated. Virtually, the contribution of some cells of the tumor stroma to the progression and metastasis is not yet understood. The tumor is a complex structure and it is now clear that stromal cells in the tumor microenvironment play an important role in cancer development (4). These could bring new insight concerning tumorigenesis and be used for the development of new therapeutic targets (4).

Mast cells (MCs) represent a controversial component of the breast cancer stromal compartment. Studies have shown that mast cells accumulate in a variety of malignant tumors, such as melanoma, Hodgkin's lymphoma and chronic lymphocytic leukemia, pancreatic, prostate and esophageal cancer (5). Some reports have shown that the high number of MCs is associated with a good prognosis (6), while others have noticed their association with poor prognosis and distant metastasis (7-9). Some reports suggest that mast cells play a promoting role in the occurrence and development of 
malignant tumors $(10,11)$. Based on the controversies found in the literature, in the present work we tried to answer whether MCs are of good or bad prognosis in breast cancer.

Based on the expression of chymase and tryptase, the mast cell-specific serine proteases, human MCs are divided into $\mathrm{MC}_{\mathrm{T}}$, which express only tryptase, and $\mathrm{MC}_{\mathrm{TC}}$, which express both enzymes (6). Preliminary investigations have suggested that during breast cancer progression, MCs may contribute to stromal remodeling and differentiation of myofibroblasts through the release of tryptase in the stromal microenvironment (11). It has been shown that MC tryptase increases cell migration and invasion and promotes breast cancer invasion (6). MCs also take part in the inflammatory reaction occurring at the periphery of the tumor (12). Mast cell-secreted angiogenic cytokines facilitate tumor vascularization not only directly but also by stimulating other inflammatory cells of the tumor microenvironment to release other angiogenic mediators (12).

Since the literature displays contradictory results regarding the role of MCs in breast cancer, the purpose of this study was to analyze how MCs' density and distribution relates with the main molecular types of breast cancer.

\section{Materials and Methods}

We investigated 80 cases of breast cancer from patients ranging between 37 and 84 years, mean of age being $66,04 \pm 8,65$ years (Table I). Patients didn't go under radio- or chemotherapy before surgery. The study was approved by the Ethics Committee of Nicolae Testemitanu State University of Medicine and Pharmacy, Chişinau, Republic of Moldova (no. 33/ 37/ 12.02.2018).

Histology. Routinely processed and paraffin embedded specimens were used for creation of tissue microarrays (TMA) by means of TMA Grand Master (3DHISTECH Ltd., Budapest, Hungary). Tissue microarrays are paraffin blocks produced by extracting cylindrical tissue cores from different paraffin donor blocks and re-embedding them into a single recipient (microarray) block (13). Sections from these blocks were stained automatically by Leica Autostainer XL (Leica Biosystems, Newcastle UponTyne, UK) and were mounted automatically (Leica CV5030, Leica Biosystems, Newcastle UponTyne, UK). Tumor histology was reviewed by 3 pathologists and appropriate sections were selected for immunohistochemical stains.

Immunohistochemistry. Immunohistochemical staining was performed automatically using Leica Bond-Max (Leica Biosystems, Newcastle UponTyne, UK). Antigen retrieval was achieved using the Bond Epitope Retrieval Solution 2 (pH 9) (Leica Biosystems, Newcastle UponTyne, UK). We used the following antibodies to establish the molecular subtype and to identify MCs: ER - clone 6F11, PR - clone 16, CK5 - clone XM26, MCT (mast cell tryptase) - clone 10D11 (all antibodies were pre-diluted, source - Leica Bond, Leica Biosystems, Newcastle UponTyne, UK); HER2 - clone CB11, EGFR - clone EGFR.113 (both pre-diluted, source - Novocastra, Leica Biosystems, Newcastle UponTyne, UK). DAB (3, 3'diaminobenzidine) (Leica Biosystems, Newcastle UponTyne, UK) was applied as a chromogen substrate and Mayer's hematoxylin was used for nuclear counterstaining.
Table I. Patients' data.

\begin{tabular}{lcccc}
\hline & \multicolumn{4}{c}{ Molecular subtype } \\
\cline { 2 - 5 } & Luminal A & Luminal B & HER2 ${ }^{+}$ & Triple-negative \\
\hline N of cases & 14 & 46 & 9 & 11 \\
Age median & 73.5 & 67.0 & 59.0 & 64.0 \\
Tumor's grade & & & & \\
G1 & 1 & 0 & 0 & 0 \\
G2 & 9 & 29 & 3 & 4 \\
G3 & 4 & 17 & 6 & 7 \\
\hline
\end{tabular}

Table II. Mean \pm standard deviation values of intra- and peritumoral MCs.

\begin{tabular}{lcc}
\hline Subtypes & MCit & MCpt \\
\hline Luminal A & $4.45 \pm 5.65$ & $9.95 \pm 8.57$ \\
Luminal B & $4.34 \pm 6.33$ & $15.66 \pm 14.54$ \\
HER2 & $1.34 \pm 1.85$ & $5.22 \pm 3.62$ \\
Triple-negative & $0.88 \pm 0.82$ & $6.72 \pm 6.24$ \\
\hline
\end{tabular}

MCit: Intratumoral mast cells; MCpt: peritumoral mast cells.

Methods of quantification. Hormone receptors (ER-estrogen receptor and $P R$-progesterone receptor) were evaluated according to Allred score (14). HER2 (human epidermal growth factor receptor 2) protein was appreciated according to the recommendations of the American Society of Clinical Oncology (15).

Quantification of brown-stained MCs (they should show a moderate to strong cytoplasmic staining) was done using the Axio Imager A2 microscope (Carl Zeiss, Germany). Sections were initially analyzed at $\times 100$ magnification in order to identify the area of highest MCs densities. We also analyzed the distribution of MCs in the tumor and peritumoral areas by counting the number of MCs from intratumoral and peritumoral stroma on 3 microscopic fields for each case at $\times 400$ magnification. The arithmetic average of the three fields was the final result for MCs in each case. Morphology of MCs was analyzed in terms of shape and granulated/ degranulated appearance.

Data analysis. A MS Excel 2010 database was used to store the data that were statistically analyzed using the SPSS statistical software package (SPSS Statistics 23.0; IBM, Chicago, IL, USA). We used Pearson's correlation coefficient (r), Spearman's rank correlation coefficient $\left(\mathrm{r}_{\mathrm{s}}\right)$ and considered a $p$-value of less than 0.05 as statistically significant.

\section{Results}

Most tumors (45 cases out of $80,56.3 \%$ ) were moderately differentiated (G2), 34 cases $(42,5 \%)$ were poorly differentiated (G3) and only 1 case $(1,3 \%)$ had a high grade of differentiation (G1). We identified the following histological types of breast 

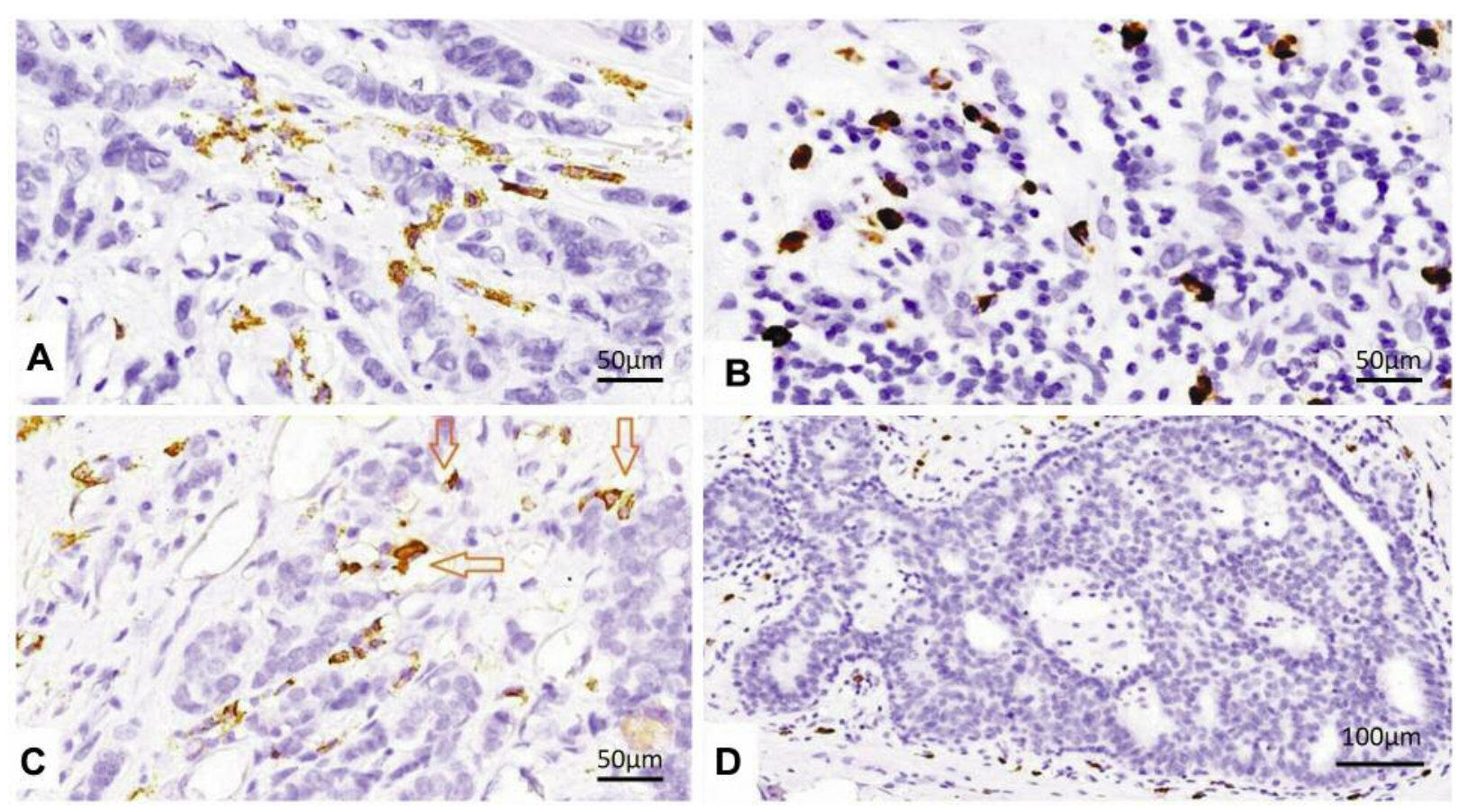

Figure 1. Mast cells (MCs) in breast carcinomas stained for anti-tryptase (brown) and, counterstained with Mayer's hematoxylin (purple). Different patterns of MCs: degranulated (A) and granulated (B). MCs in proximity to lymphatic vessels (arrows) (C). MCs surrounding the tumor nest (D). Scale bars: $A-C=50 \mu \mathrm{m}, D=100 \mu \mathrm{m}$.

carcinoma: i) ductal invasive in 74 cases $(92.5 \%)$, ii) lobular invasive in 3 cases $(3.75 \%)$, iii) lobular in situ in 2 cases (2.5\%), and iv) ductal in situ in 1 case (1.25\%).

Immunohistochemical staining revealed: i) 46 cases of luminal B subtype $(57.5 \%)$, ii) 14 cases of luminal A $(17.5 \%)$, iii) 9 cases of HER2+ $(11.3 \%)$ and iv) 11 cases $(13.8 \%)$ of triple-negative breast carcinomas.

Brown stained MCs were identified in all the slides. Most of them were oval- or spindle-shaped and appeared either as granulated or degranulated (Figure 1A and B). The degranulated MCs possessed an irregular shape with an uneven color and a non-complete cell membrane surface, while the granulated MCs were round or oval with a uniform color and an intact cell membrane surface.

We noticed a prevalence of MCs in the region of lymphatic vessels (Figure 1C). Evaluation of slides pointed out that MCs prevail in the peritumoral stroma, with a tendency to surround the tumor nest, as shown in Figure 1D. The mean of MCpt was $12.2 \pm 12.5$ cells, with a maximum of 65 cells, while the mean of MCit was $3.5 \pm 5.5$ cells, with a maximum of 27.6 cells.

Highest values of MCs were determined in the luminal B subtype (mean and standard deviation being $4.34 \pm 6.33$ for intratumoral and $15.66 \pm 14.54$ for peritumoral MCs). These were followed by the luminal A subtype (mean and standard deviation being $4.45 \pm 5.65$ and $9.95 \pm 8.57$ for the intra- and peritumoral MCs respectively) (Table II). For Luminal A, a partial correlation was detected between MCit and PR $(\mathrm{rs}=0.703, p=0.005)$. Luminal B subtype also revealed a positive correlation between MCit and MCpt $(\mathrm{r}=0.446$, $p=0.002$ ) and a positive correlation between MCpt and hormone receptors (ER: $\mathrm{r}=0.351, p=0.017$ and PR: $\mathrm{r}=0.311$, $p=0.035$ ). MCpt positively correlated with the patients' age $(\mathrm{r}=0.382, p=0.009)$, as well as with HER ${ }^{+}$carcinoma cells $(\mathrm{r}=0.680, p=0.044)$ in HER $2^{+}$subtype, while both MCit and MCpt positively correlated with one another in triplenegative breast carcinomas $(\mathrm{r}=0.825, p=0.002)$.

Analyzing overall molecular subtypes, we found a positive correlation between MCit and MCpt ( $\mathrm{r}=0.442, p=0.000041)$. In G2 tumors, MCpt correlated with ER+ carcinoma cells $(\mathrm{r}=0.360, p=0.015)$ (Table III) while in G3 tumors ER correlated with both MCit and MCpt ( $\mathrm{r}=0.439, p=0.009$ and $\mathrm{r}=0.566, p=0.000487$, respectively) (Table IV).

\section{Discussion}

Mast cells (MCs) were first identified in human tumors and were named as such by Paul Ehrlich (16). These cells store and release a variety of biologically active substances that can affect various target cells. Although usually associated with allergic disorders, MCs are a major source of pro-tumorigenic (e.g., angiogenic and lymphangiogenic factors) and anti- 
Table III. Correlations and p-Values for G2 tumors.

\begin{tabular}{|c|c|c|c|c|c|c|c|c|}
\hline & & HistoType & Grade & ER & PR & HER 2 & MCit & $\mathrm{MCpt}$ \\
\hline \multirow[t]{3}{*}{ HistoType } & $r$ & 1 & & 0.086 & 0.201 & -0.142 & -0.092 & 0.022 \\
\hline & $\mathrm{p}$ & & & 0.574 & 0.186 & 0.351 & 0.548 & 0.885 \\
\hline & $\mathrm{N}$ & 45 & 45 & 45 & 45 & 45 & 45 & 45 \\
\hline \multirow[t]{3}{*}{ Grade } & $r$ & & & & & & & \\
\hline & $\mathrm{p}$ & & & & & & & \\
\hline & $\mathrm{N}$ & 45 & 45 & 45 & 45 & 45 & 45 & 45 \\
\hline \multirow[t]{3}{*}{ ER } & $r$ & 0.086 & & 1 & 0.584 & 0.064 & 0.290 & 0.360 \\
\hline & $\mathrm{p}$ & 0.574 & & & 0.000025 & 0.675 & 0.053 & 0.015 \\
\hline & $\mathrm{N}$ & 45 & 45 & 45 & 45 & 45 & 45 & 45 \\
\hline \multirow[t]{3}{*}{ PR } & $\mathrm{r}$ & 0.201 & & 0.584 & 1 & 0.127 & 0.042 & 0.310 \\
\hline & $\mathrm{p}$ & 0.186 & & 0.000025 & & 0.405 & 0.782 & 0.038 \\
\hline & $\mathrm{N}$ & 45 & 45 & 45 & 45 & 45 & 45 & 45 \\
\hline \multirow[t]{3}{*}{ HER 2} & $\mathrm{r}$ & -0.142 & & 0.064 & 0.127 & 1 & -0.041 & 0.267 \\
\hline & $\mathrm{p}$ & 0.351 & & 0.675 & 0.405 & & 0.791 & 0.076 \\
\hline & $\mathrm{N}$ & 45 & 45 & 45 & 45 & 45 & 45 & 45 \\
\hline \multirow[t]{3}{*}{ MCit } & $\mathrm{r}$ & -0.092 & & 0.290 & 0.042 & -0.041 & 1 & 0.423 \\
\hline & $\mathrm{p}$ & 0.548 & & 0.053 & 0.782 & 0.791 & & 0.004 \\
\hline & $\mathrm{N}$ & 45 & 45 & 45 & 45 & 45 & 45 & 45 \\
\hline \multirow[t]{3}{*}{ MCpt } & $\mathrm{r}$ & 0.022 & & 0.360 & 0.310 & 0.267 & 0.423 & 1 \\
\hline & $\mathrm{p}$ & 0.885 & & 0.015 & 0.038 & 0.076 & 0.004 & \\
\hline & $\mathrm{N}$ & 45 & 45 & 45 & 45 & 45 & 45 & 45 \\
\hline
\end{tabular}

R: Pearson's correlation coefficient; p: p-value; N: number of cases; HistoType: histological type of tumor; Grade: tumor's grade; MCit: intratumoral MCs; MCpt: peritumoral MCs.

tumorigenic molecules (e.g., TNF- $\alpha$ and IL-90), thus, their role on breast cancer is controversial (5). Some scholars believe that mast cell infiltration suggests a good prognosis of breast cancer: similar to our results, three other groups have observed high MCs density in luminal A and B types of breast cancer, which can be hormonally treated and have a better prognosis $(6,10,17)$. This suggests that MCs are associated with less aggressive tumors. On the other hand, we know that estrogen acts as a proliferative factor, and can stimulate breast cancer development (18). This can explain why in our study both MCit and MCpt interrelated with ER in G3 tumors. Positive correlations between ER and MCs suggest that estrogen is a chemotactic molecule for MCs. We were especially interested in the correlation between MCit and $\mathrm{ER}^{+}$carcinoma cells, as some studies have proved that estrogen activates mast cells in ovarian endometriosis and that human uterine mast cells express $\operatorname{ER} \beta(19,20)$. Others have shown that administration of estrogens leads to eosinophilic and MCs infiltrations (21). MCs play a pro-tumorigenic role in human bladder cancer through stimulating ER $\beta$ and it has been demonstrated in a murine model of bladder cancer that a selective ER $\beta$ antagonist can inhibit mast cell-promoted tumor growth (9).

Other groups, however, have shown a negative correlation between populations of MCs and tumor grade (6), data that was not confirmed by our study.

Some groups have concluded that the prognosis is worse with a higher density of mast cells in the breast cancer tissue (10). Xiang et al. have observed more numerous peritumoral MCs in G3 breast cancers, increased tryptase being associated with higher tumor grade and more lymph node metastasis compared to lower grades. They have also noted that tryptase promotes the invasion and migration of breast cancer cells along with the activation of matrix metalloproteinase-2, and have concluded that tryptase promotes breast cancer migration and invasion (23). Raica et al. revealed strong positive correlations between populations of MCs and lymphatic vessels in some molecular subtypes of breast cancer, thus supporting the idea of MCs involvement in metastasis by lymphangiogenesis (11). Ribatti et al. have pointed out that angiogenesis increased in parallel with the number of tryptase-positive MCs particularly inside lymph nodes associated with micrometastases compared to non-metastatic lymph nodes (24). It has also been demonstrated that during breast cancer progression MCs may contribute to stromal remodeling and differentiation of myofibroblasts, through tryptase released in the stromal microenvironment (25). All these mean that targeting MCs could be involved in the inhibition of angiogenesis, lymphangiogenesis and many other negative effects of MCs' activation.

MCs' dynamics is strongly influenced by hormone receptors and HER2 status in breast cancer. Our findings suggest that MCit increased especially in aggressive tumor types and serves as a worse prognostic factor. 
Table IV. Correlations and p-Values for G3 tumors.

\begin{tabular}{|c|c|c|c|c|c|c|c|c|}
\hline & & HistoType & Grade & ER & PR & HER2 & MCit & $\mathrm{MCpt}$ \\
\hline \multirow[t]{3}{*}{ HistoType } & $\mathrm{r}$ & 1 & & 0.166 & 0.484 & 0.364 & -0.154 & 0.130 \\
\hline & $\mathrm{p}$ & & & 0.349 & 0.004 & 0.035 & 0.386 & 0.465 \\
\hline & $\mathrm{N}$ & 34 & 34 & 34 & 34 & 34 & 34 & 34 \\
\hline \multirow[t]{3}{*}{ Grade } & $\mathrm{r}$ & & & & & & & \\
\hline & $\mathrm{p}$ & & & & & & & \\
\hline & $\mathrm{N}$ & 34 & 34 & 34 & 34 & 34 & 34 & 34 \\
\hline \multirow[t]{3}{*}{ ER } & $\mathrm{r}$ & 0.166 & & 1 & 0.572 & 0.139 & 0.439 & 0.566 \\
\hline & $\mathrm{p}$ & 0.349 & & & 0.000404 & 0.434 & 0.009 & 0.000487 \\
\hline & $\mathrm{N}$ & 34 & 34 & 34 & 34 & 34 & 34 & 34 \\
\hline \multirow[t]{3}{*}{ PR } & $\mathrm{r}$ & 0.484 & . & 0.572 & 1 & 0.397 & 0.319 & 0.572 \\
\hline & $\mathrm{p}$ & 0.004 & & 0.000404 & & 0.020 & 0.066 & 0.000406 \\
\hline & $\mathrm{N}$ & 34 & 34 & 34 & 34 & 34 & 34 & 34 \\
\hline \multirow[t]{3}{*}{ HER2 } & $\mathrm{r}$ & 0.364 & & 0.139 & 0.397 & 1 & 0.028 & 0.105 \\
\hline & $\mathrm{p}$ & 0.035 & & 0.434 & 0.020 & & 0.875 & 0.553 \\
\hline & $\mathrm{N}$ & 34 & 34 & 34 & 34 & 34 & 34 & 34 \\
\hline \multirow[t]{3}{*}{ MCit } & $\mathrm{r}$ & -0.154 & & 0.439 & 0.319 & 0.028 & 1 & 0.570 \\
\hline & $\mathrm{p}$ & 0.386 & & 0.009 & 0.066 & 0.875 & & 0.000430 \\
\hline & $\mathrm{N}$ & 34 & 34 & 34 & 34 & 34 & 34 & 34 \\
\hline \multirow[t]{3}{*}{ MCpt } & $\mathrm{r}$ & 0.130 & & 0.566 & 0.572 & 0.105 & 0.570 & 1 \\
\hline & $\mathrm{p}$ & 0.465 & & 0.000487 & 0.00040 & 0.553 & 0.00043 & \\
\hline & $\mathrm{N}$ & 34 & 34 & 34 & 34 & 34 & 34 & 34 \\
\hline
\end{tabular}

R: Pearson's correlation coefficient; p: p-value; N: number of cases; HistoType: histological type of tumor; Grade: tumor's grade; MCit: intratumoral MCs; MCpt: peritumoral MCs.

\section{Conflicts of Interest}

The authors declare that the research was conducted in the absence of any commercial or financial relationship that could be construced as a potential conflict of interest.

\section{Authors' Contributions}

EC collected the data, interpreted the data, wrote the paper. RAC collected data, wrote the paper. AMC interpreted the data, wrote the paper. PNG collected the data. LS, VF and VD performed the statistical analysis. MR conceived and coordinated this study and wrote the paper.

\section{Acknowledgements}

This work was supported by the CNFIS-FDI-2018-0459 grant offered by the Romanian Ministry of Education and Research.

\section{References}

1 Bray F, Ferlay J and Soerjomataram I: Global Cancer Statistics 2018: GLOBOCAN Estimates of Incidence and Mortality Worldwide for 36 Cancers in 185 Countries. CA Cancer J Clin 68: 394-424, 2018. PMID: 30207593. DOI: 10.3322/caac.21492

2 Prat A and Perou CM: Deconstructing the molecular portraits of breast cancer. Mol Oncol 5(1): 5-23, 2011. PMID: 21147047. DOI: $10.1016 /$ j.molonc.2010.11.003
3 Bense RD, Sotiriou C, Piccart-Gebhart MJ, Haanen JBAG, Van Vugt MATM, De Vries EGE, Schroder CP and Fehrmann RSN: Relevance of tumor-infiltrating immune cell composition and functionality for disease outcome in breast cancer. J Natl Cancer Inst 109(1): 1-9, 2017. PMID: 27737921. DOI: 10.1093/jnci/ djw192

4 Mao Y, Keller ET, Garfield DH, Kunwei Shen A and Wang J: Stromal cells in tumor microenvironment and breast cancer. Cancer Metastasis Rev 32(0): 303-315, 2013. PMID: 23114846. DOI: $10.1007 / \mathrm{s} 10555-012-9415-3$

5 Varricchi G, Galdiero MR, Loffredo S, Marone G, Iannone R, Marone G and Granata F: Are mast cells MASTers in cancer? Front Immunol 8(424): 1-13, 2017. PMID: 28446910. DOI: 10.3389/fimmu.2017.00424

6 Glajcar A, Szpor J, Pacek A, Tyrak KE, Chan F, Streb J, Hodorowicz-Zaniewska D and Okoń K: The relationship between breast cancer molecular subtypes and mast cell populations in tumor microenvironment. Virchows Arch 470(5): 505-515, 2017. PMID: 28315938. DOI: 10.1007/s00428-017-2103-5

7 Li L, Dang Q, Xie H, Yang Z, He D, Liang L, Song W, Yeh S and Chang $C$ : Infiltrating mast cells enhance prostate cancer invasion via altering LncRNA-HOTAIR/PRC2-androgen receptor (AR)-MMP9 signals and increased stem/progenitor cell population. Oncotarget 6(16): 14179-14190, 2015. PMID: 25895025. DOI: $10.18632 /$ oncotarget.3651

8 Öhrvik H, Grujic M, Waern I, Gustafson A-M, Ernst N, Roers A, Hartmann K and Pejler G: Mast cells promote melanoma colonization of lungs. Oncotarget 7(42): 68990-69001, 2016. PMID: 27602499. DOI: 10.18632/oncotarget.11837 
9 Rao Q, Chen Y, Yeh C-R, Ding J, Li L, Chang C and Yeh S: Recruited mast cells in the tumor microenvironment enhance bladder cancer metastasis via modulation of ER $\beta /$ CCL2/CCR2 EMT/MMP9 signals. Oncotarget 7(7): 20-22, 2016. PMID: 26556868. DOI: 10.18632 /oncotarget.5467

10 Sang J, Yi D, Tang X, Zhang Y and Huang T: The associations between mast cell infiltration, clinical features and molecular types of invasive breast cancer. Oncotarget 7(49): 81661-81669, 2016. PMID: 27835573. DOI: 10.18632/oncotarget.13163

11 Raica M, Cimpean AM, Ceauşu R, Ribatti D and Gaje P: Interplay between mast cells and lymphatic vessels in different molecular types of breast cancer. Anticancer Res 33(3): 957-964, 2013. PMID: 23482767.

12 Cimpean AM, Tamma R, Ruggieri S, Nico B, Toma A and Ribatti D: Mast cells in breast cancer angiogenesis. Crit Rev Oncol Hematol 115: 23-26, 2017. PMID: 28602166. DOI: 10.1016/j.critrevonc.2017.04.009

13 Jawhar NMT: Tissue Microarray: A rapidly evolving diagnostic and research tool. Ann Saudi Med 29(2): 123-127, 2009. PMID: 19318744. DOI: $10.4103 / 0256-4947.51806$

14 Allred C, Harvey JM, Berardo M and Clark GM: Prognostic and predictive factors in breast cancer by immunohistochemical analysis. Mod Pathol 11: 155-168, 1998. PMID: 9504686

15 Wolff AC, Hammond MEH, Allison KH, Harvey BE, Mangu PB, Bartlett MS, Bilous M, Ellis IO, Fitzgibbons P, Hanna W, Jenkins RB, Press MF, Spears PA, Vance GH, Viale G, Mcshane LM and Dowsett M: Human epidermal growth factor receptor 2 testing in breast cancer: American Society of Clinical Oncology/College of American Pathologists Clinical Practice Guideline Focused Update. J Clin Oncol 36(20): 2105-2122, 2019. PMID: 29846122. DOI: 10.1200/JCO.2018.77.8738

16 Ehrlich P: Beiträge zur Kenntniss der granulirten Bindegewebszellen und der eosinophilen Leukocythen. Arch Anat Physiol 3: 166-169, 1879.

17 Della Rovere F, Granata A, Familiari D, D’Arrigo G, Mondello B and Basile G: Mast cells in invasive ductal breast cancer: Different behavior in high and minimum hormone-receptive cancers. Anticancer Res 27(4 B): 2465-2471, 2007. PMID: 17695540

18 Russo J, Russo IH. The role of estrogen in the initiation of breast cancer. J Steroid Biochem Mol Biol 102(1-5): 89-96, 2006. PMID: 17113977. DOI: 10.1016/j.jsbmb.2006.09.004
19 Zhu T, Shao-Jie D, TIan-Tian L, Li-Bo Z, Xiu-Feng H and XinMei Z: Estrogen is an important mediator of mast cell activation in ovarian endometriomas. Reproduction 155(1): 73-83, 2016. PMID: 29074615. DOI: 10.1530/REP-17-0457

20 De Leo B, Esnal-Zufiaurre A, Collins F, Critchley HOD and Saunders PTK: Immunoprofiling of human uterine mast cells identifies three phenotypes and expression of ER $\beta$ and glucocorticoid receptor. F1000Res 6(667): 1-19, 2017. PMID: 28620462. DOI: 10.12688/f1000research.11432.2

21 Mocan-Hognogi RF, Costin N, Malutan A, Ciortea R, Trif IA, Nagy AL, Bogdan ML and Mihu D: Histological changes in the vulva and vagina from ovariectomised rats undergoing oestrogen treatment. Folia Morphol 75(4): 467-473, 2016. PMID: 27830882. DOI: 10.5603/FM.a2016.0026

22 Austin D, Hamilton N, Elshimali Y, Pietras R, Wu Y and Vadgama $\mathrm{J}$ : Estrogen receptor-beta is a potential target for triple negative breast cancer treatment. Oncotarget 9(74): 33912-33930, 2018. PMID: 30338035. DOI: 10.18632/oncotarget.26089

23 Xiang M, Gu Y, Zhao F, Lu H, Chen S and Yin L: Mast cell tryptase promotes breast cancer migration and invasion. Oncol Rep 23(3): 615-619, 2010. PMID: 20126998. DOI: 10.3892 /or_00000676

24 Ribatti D, Finato N, Crivellato E, Guidolin D, Longo V, Mangieri D, Nico B, Vacca A and Beltrami CA: Angiogenesis and mast cells in human breast cancer sentinel lymph nodes with and without micrometastases. Histopathology 51(6): 837-842, 2007. PMID: 17944928. DOI: 10.1111/j.1365-2559.2007. 02869.x

25 Mangia A, Malfettone A, Rossi R, Paradiso A, Ranieri G, Simone $G$ and Resta L: Tissue remodelling in breast cancer: human mast cell tryptase as an initiator of myofibroblast differentiation. Histopathology 58(7): 1096-1106, 2011. PMID: 21707711. DOI: $10.1111 / \mathrm{j} .1365-2559.2011 .03842 . x$ 